Chapter 4

\title{
Dental-Related Stem Cells and Their Potential in Regenerative Medicine
}

\author{
Razieh Karamzadeh and \\ Mohamadreza Baghaban Eslaminejad \\ Additional information is available at the end of the chapter \\ http://dx.doi.org/10.5772/55927
}

\section{Introduction}

Stem cells have been opening a promising future in clinical therapies because of their two remarkable features known as self-renewal and multi-lineage differentiation. These cells can be classified in terms of their origin (embryonic, prenatal and postnatal stem cells) as well as the differentiation commitments (pleuripotent, multipotent and unipotent). Postnatal stem cells, also known as the adult stem cells (ASCs), normally exist in almost every adult tissues, including bone marrow, skin, neural tissues, and dental epithelium, acting as supportive cells by their regeneration capacity. Among different stem cell types, ASCs seem to be more applicable in stem cell-mediated therapies and regenerative medicine because these cells lack ethical concerns, and possesses less tumorgenic potency than their embryonic counterparts.

Recently, human dental stem cells (DSCs), a subtype of ASCs, have drawn worldwide attention for future therapies due to their both technical and practical superiorities. In addition to having some mesenchymal stem cell (MSC) characteristics, including plastic adherent ability with formation of colonies in vitro, and also immunoprivileged properties, DSCs are easilyaccessible cells with higher proliferation capacity than ordinary marrow-derived MSCs. Currently, there are six types of stem/progenitor cells determined in dental-related tissues. 1) dental pulp stem cell (DPSCs), 2) stem cells from human exfoliated deciduous teeth (SHED), 3) periodontal ligament stem cells (PDLSC), 4) stem cells from apical papilla (SCAP) of developing tooth, 5) dental follicle stem/progenitor cells (DFPCs) and 6) gingiva stem cells (GSCs). DPSCs, SHEDs and SCAPs are referred to as dental pulp-related stem cells, and PDLSCs \& DFPCs as periodontium-related stem cells [1, 5]. 
This chapter focuses on different aspects of dental-derived adult stem cells, such as their classification, biological characterization, initiating culture, cultivation systems, cryopreservation and potential applications in tissue engineering and regenerative medicine. The data are organized as three main parts, including: 1) Dental-related stem cell biology: from the classification to the characterization and differentiation potential 2) Dental-related stem cell initiation culture, culture systems and cryopreservation 3) Dental-related stem cell- based tissue regeneration.

\section{Dental-related stem cell biology: From the classification to the characterization \& differentiation potential}

According to the literature, there are several types of stem/progenitor cells existed in dental tissue. In this section, each of these cells will be described in terms of their main characteristics.

\subsection{Dental Pulp Stem Cells (DPSCs)}

The presence of stem cells in dental pulp tissue primarily have been reported in 1985 by Yamamura [3, 4, 6] (Figure 1). Later on, Caplan et al. have demonstrated that these cells presented osteogenic and chondrogenic potential in vitro, and could also differentiate into dentin, in vivo. In 2000, Gronthos et al. have isolated dental pulp stem cells from adult human dental pulp, which had the ability to regenerate a dentin-pulp-like complex [7]. Interestingly, some recent works have found the presence of stem cells in inflamed pulp with capacity to form mineralized matrix both in vitro and in vivo. These findings make dental pulp as an interesting tissue source of putative stem cells, even in diseased form.[8]

DPSCs are similar to MSCs in some ways: they are of fibroblastic morphology with selective adherence to solid surfaces, having good proliferative potential and capacity to differentiate in vitro, and the ability to repair tissues in vivo. It's interesting to note that DPSCs could differentiate into not only osteoblasts, chondrocytes \& adipocytes, but also myocytes, neurons and hepatocytes lineages in vitro [4]. DPSCs are characterized by their negative expression of hematopoietic antigens (e.g., CD45, CD34, CD14), and positive expression of stromal-associated markers (e.g., CD90, CD29, CD73, CD105, CD44) (Table 1). They also express multipotent marker (STRO-1) and extracellular matrix proteins, such as collagen, vimentin, laminin, and fibronectin. [9-11]. Interestingly, some of the pluripotent stem cell markers, such as Oct4, Nanog, Sox2, Klf4, SSEA4 \& c-Myc have been reported to express on DPSCs [12-14]. More recently, it was demonstrated that core transcription factor of the reprogramming Oct4, Nanog, Klf4 and c-Myc become significantly down-regulated following the DPSC differentiation [4].

Apart from stemness markers, DPSCs are also shown to express bone markers, such as bone sialoprotein, osteocalcin, alkaline phosphates (ALP), and type I collagen. This indicates their differentiation commitment into bone tissue [15]. On the other hand, the expression of dentin sialophosphoprotein (an odontoblast specific protein precursor) is not present in the cultures of hDPSCs implied that these cells represent an undifferentiated pre-odontogenic phenotype $[7,16]$. 
From immunological perspective, it has been reported that DPSCs displayed more immnosuppressive activities than the BM-MSCs. This was obvious in inhibiting $\mathrm{T}$ cells response in vitro [17].

Based on some investigations, there is a sub-type of DPSCs referred to as "immature dental pulp stems cells" (IDPSCs), which have promising potential in future stem cell researches. IDPSCs were firstly, isolated from pulp tissue of the human exfoliated deciduous as well as permanent teeth [18]. These cells express both embryonic and MSC markers (see part 2.2). It has been indicated that transferring of human IDPSCs (hDPSCs) into mouse blastocysts resulted in formation of human/mouse chimera which was able to retain proliferation and differentiation capacity [19]. Furthermore, hIDPSCs possess the capacity to rapidly reprogrammed into induced pleuripotent stem cells (iPSc) which are able to produce primary hIDPSC-iPSC colonies even under feeder-free conditions [20].

\subsection{Dental Stem cells from Human Exfoliated Deciduous teeth (SHED)}

In 2003, Miura et al. have reported to isolate a stem cell population from the living pulp remnants of exfoliated deciduous teeth. These authors have termed the cells as stem cells from human exfoliated deciduous teeth (SHED) [21] (Figure 1). These cells which are believed to be of the neural crest origin are heterogeneous fibroblast-like population possessing an extensive proliferating capacity than either DPSCs or BM-MSCs [22]. In terms of surface epitopes, it has been found that they express markers of MSCs (STRO-1, CD146, SSEA4, CD90, CD73, CD 105, CD106 and CD 166) and lack of hematopoietic/endothelial markers (CD34, CD31) (Table 1). Under an appropriate culture conditions, SHED are able to differentiate into the variety of cell types, including neural cells, angiogenic endothelial cells, adipocytes, osteoblasts, and odontoblasts [23-25]. In vivo transplantation of SHED have been reported to result in formation of bone and dentin like-tissue. [18, 21, 26-29]. There are some studies suggested that SHED is different from IDPSCs in terms of expression of stem cell markers (see part 1.1)[18, 30]. Moreover, some research works have been reported that SHED would possess immunomodulatory function as seen in BM-MSCs [28].

\subsection{Periodontal Ligament Stem Cells (PDLSC)}

Periodontal ligament stem cells (PDLSCs) have first been introduced by Seo et al. [31] (Figure 1). Like MSCs, PDLSCs have been reported to form adherent clonogenic population of fibroblast-like cells in the culture. They express both early MSC markers such as, STRO-1 and CD146, and other MSC and pluripotent makers, such as CD44, CD90, CD105, CD73, CD26, CD10, CD29 and CD166; meanwhile, they have no expression for CD40, CD80, and CD86[31-33] (Table 1). Some investigations have revealed that PDLSCs may be positive for embryonic stem cell markers, as well, including SSEA1, SSEA3, SSEA4, TRA-1-60, TRA-1-81, Oct4, Nanog, Sox2 and Rex1, and ALP [34]. Based on some research works, SSEA4-positive PDLSCs displayed the potential to generate adipocytes, osteoblasts, chondrocytes (from mesodermal layer), neurons (from ectodermal layer), and hepatocytes (from endodermal lineage) in vitro $[31,34,35]$. Furthermore, it has been shown that transplantation of PDLSCs 
into immunocompromised rodents resulted in the generation of cementum/PDL-like structure and contributes to periodontal tissue repair [31].

PDLSCs show immunomodulatory activity by up-regulation of soluble immunosuppressive factors (TGF- $\beta 1$, hepatocyte growth factor (HGF) and indoleamine 2, 3-dioxygenase (IDO) in the presence of activated peripheral blood mononuclear cells (PBMNCs). Similar to the DPSCs, PDLSCs are positive for HLA-ABC (MHC class I antigen) while negative for HLA-DR (MHC class II antigen) [32].

\subsection{Dental Follicle Progenitor Cells (DFPCs)}

In 2005 \& 2007, Morsczeck et al. and Kémoun et al., respectively have identified unique undifferentiated lineage committed cells possessing mesenchymal progenitor features in the human dental follicle (Figure 1). The cells were referred to as "dental follicle precursor cells" (DFPCs) [36, 37]. Characteristically, DFPCs, similar to the bone marrow stem cells, are adherent and colony-forming cells. These cells have been reported to express Notch-1, CD13, CD44, CD73, CD105, and STRO-1 [1, 36] (Table 1). Human DFPCs has been believed to consist of precursor cells for cementoblasts, periodontal ligament cells, and osteoblasts. Under appropriate in vitro conditions, they are capable of differentiating into osteoblasts, cementoblasts, chondrocytes and adipocytes. Interestingly, although both DFPCs and SHED are of the neural crest origin, their neural differentiation potentials are different under the same culture conditions. It has been reported that SHED possess good differentiation potential than DFPCs in terms of the expression of Pax6 which is a marker of retinal stem cells [27].

\subsection{Stem Cells from the Apical Papilla (SCAP)}

Stem cells from dental apical papilla (SCAP) were first identified and characterized by Sonoyama et al. in human permanent immature teeth [38] (Figure 1). These authors described the cells as adherent clonogenic cells with mesenchymal stem cell features, which are expressed STRO-1, CD24, CD29, CD73, CD90, CD105, CD106, CD146, CD166, and ALP, and not expressing CD34; CD45; CD18; and CD150. Among these markers, CD24 would be of a specific marker for SCAP since it's not found in the other dental stem cells (Figure 2). Excitingly, some authors have reported that SCAP display higher telomerase expression than both DPSCs and BMMSCs [38]. Furthermore, SCAP has been shown to positively stain with several neural markers implying their possible origin from the neural crest [39]. In terms of differentiation, SCAP are capable of generating osteoblasts, odontoblasts and adipocytes in vitro. An in vivo study has demonstrated that these cells form hard tissue when being loaded onto hydroxyapatite (HA) and implanted subcutaneously in immunocompromised rats [38-40]. Moreover, SCAP have been reported to possess a significantly higher mineralization potential as well as proliferation rate than DPSCs. This finding might be of some importance for their use in dental and/or bone tissue engineering and regeneration [41].

About the possibility of immunogenicity of SCAPs, an independent study have reported that swine SCAPs are non-immunogenic and suppressed T cells proliferation in vitro [42]. 


\subsection{Stem Cells derived from Gingiva (GSCs)}

The isolation of a stem cell population from gingiva was firstly reported by Zhang et al. in 2009 [43] (Figure 1). These authors derived the cells from the spinous layer of human gingiva and referred to them as gingival stem cells (GSCs). In terms of markers, it has been shown that GSCs are negative for CD45/CD34, but positive for CD29, CD44, CD73, CD90, CD105, CD146, STRO-1 and SSEA4 (Table 1). In addition, extracellular matrix proteins, such as collagen, vimentin, Collagen type-1, and fibronectin have been reported to express in these cells [43, 44]. Like MSCs, GSCs possess a differentiation potential into osteoblasts, adipocytes and chondrocytes in vitro [45]. Moreover, these cells have been found to be able to differentiate along endothelial as well as neural cell lineages. Furthermore, in vivo bone regeneration potential of GSCs was demonstrated by transplantation of GSCs/HA into immunocompromised mice [45]. More importantly, in a comparative study, it was demonstrated that GSCs showed stable phenotypes, maintain normal karyotype and telomerase activity in long-term cultures in comparison with BM-MSC [45].

As with other dental related stem cells, GSCs has been found to display immunomodulatory functions; they inhibit lymphocytes proliferation and express a wide range of immunosuppressive factors, including Interleukin-10 (IL-10), IDO, inducible NO synthase (iNOS), and cyclooxygenase 2 (COX-2) in response to the inflammatory cytokine, IFN- $\gamma$ [43].

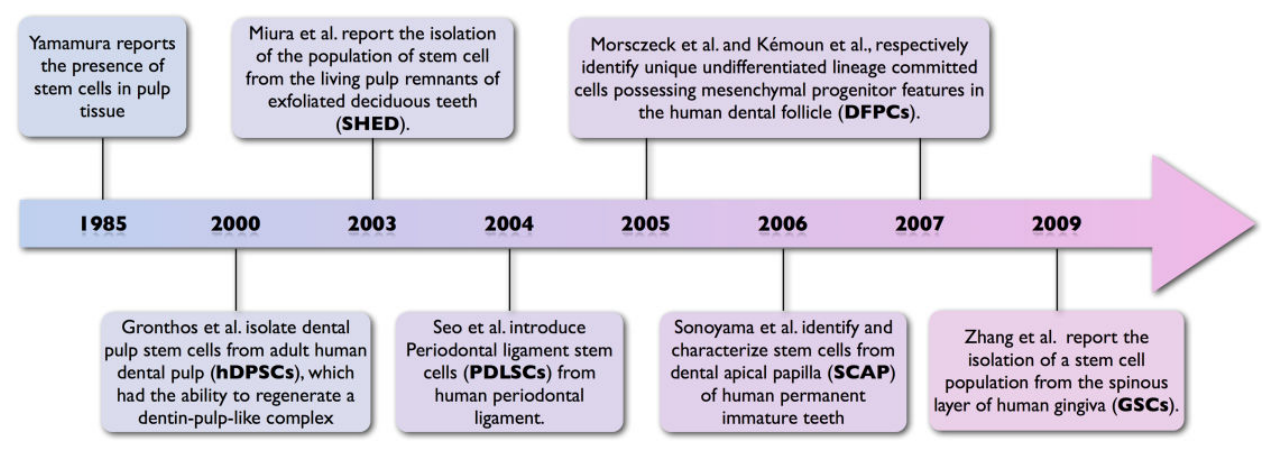

Figure 1. Timeline about the highlights in the history of the isolation of dental-related stem cells (see text). 


\begin{tabular}{|c|c|c|c|c|c|c|}
\hline & DPSCs & SHED & PDLSCs & DFPCs & SCAPs & GSCs \\
\hline \multirow[t]{15}{*}{$\mathrm{CD}(+)$} & STRO-1 & STRO-1 & STRO-1 & STRO-1 & STRO-1 & STRO-1 \\
\hline & CD10 & & CD10 & CD10 & & \\
\hline & CD13 & CD13 & CD13 & CD13 & CD13 & CD13 \\
\hline & & & & & CD24 & \\
\hline & & & CD26 & & & \\
\hline & CD29 & CD29 & CD29 & CD29 & CD29 & CD29 \\
\hline & CD44 & CD44 & CD44 & CD44 & CD44 & CD44 \\
\hline & CD59 & & CD59 & CD59 & & \\
\hline & CD73 & CD73 & CD73 & CD73 & CD73 & CD73 \\
\hline & CD90 & CD90 & CD90 & CD90 & CD90 & CD90 \\
\hline & CD105 & CD105 & CD105 & CD105 & CD105 & CD105 \\
\hline & CD106 & CD106 & CD106 & & CD106 & CD106 \\
\hline & CD117 & & & & & \\
\hline & CD146 & CD146 & & & CD146 & CD146 \\
\hline & & CD166 & CD166 & & CD166 & CD166 \\
\hline \multirow[t]{11}{*}{$C D(-)$} & CD14 & CD14 & CD14 & & & \\
\hline & & & & & CD18 & \\
\hline & CD19 & & & & & \\
\hline & CD24 & & & & & \\
\hline & CD34 & CD34 & CD34 & CD34 & CD34 & CD34 \\
\hline & & & CD40 & & & \\
\hline & CD45 & CD45 & CD45 & CD45 & CD45 & CD45 \\
\hline & & & CD80 & & & \\
\hline & & & CD86 & & & \\
\hline & & & & & CD150 & \\
\hline & HLA-DR & & HLA-DR & HLA-DR & & HLA-DR \\
\hline
\end{tabular}

Table 1. Cell Surface Marker Profiles of dental-related stem cells. DPSC; Dental pulp stem cell, SHED; Stem cells from human exfoliated deciduous teeth, PDLSC; Periodontal ligament stem cells, DFPC, Dental follicle precursor cells, SCAP; Stem cells from dental apical papilla, GSC; Gingival stem cells. [1-4]

\section{Dental-related stem cell initiation culture, culture systems and cryopreservation}

In dental related stem cell researches, the first step is to isolate cells from tissue sources. The next step is to expand the cells into sufficient number. In some occasion, it may be necessary to preserve the cells for future use since tissue sources would not be available on demand. In this section, we will describe the location of the certain teeth tissue from which the stem cell population can be derived and followed by description of common methods by which the isolation culture can be initiated. At the end, culture systems for the cell propagation as well as the main points regarding issue of cell cryopreservation will be explained. 


\subsection{Anatomical location of teeth tissue from which DSCs can be derived}

Most of the human DSCs are come from teeth, which are subjected to the orthodontic treatments. Based on the studies, molars and premolars are mostly used for this purpose. Third molars (wisdom teeth) are the most common teeth for extraction in dental clinical practice. In addition, developing wisdom teeth during the adult life are the excellent candidates as the accessible source of developing tissue similar to those in embryonic development. There are a few studies considering the supernumerary teeth derived from other teeth, such as canine, for the isolation of DPSCs [12] In some cases, such as the isolation of DPSCs from inflamed dental pulps, endodontic treatments are used rather than orthodontics surgeries [46]. In any case, considering the precise location of the dental tissues in tooth anatomy is important to achieve certain types of DSCs with minimum cell contaminations. Hence, here is the brief description of the localization for the specific DSCs isolation.

\subsubsection{Tissues contained dental pulp-related stem cells (DPSCs, SHED \& SCAP)}

Isolation of DPSCs following the pulp extraction could be achieved by either through the root or crown of the dental organ. In the case of SHEDs or DPSCs, which are derived from incompleted root teeth, the exposed pulp is accessible from the root without applying any specific procedure [21]. In other situation (extracted permanent or deciduous teeth), dental pulp extraction is accomplished through the dental crown by cutting the cementum-enamel junction (CEJ) using dental instruments, such as pliers (bone forceps), extirpation needle, Gracey curette, dental fissure burs, etc. [6] Moreover, in the case of inflamed teeth, pulp tissues are removed during the endodontic therapies [46] (Figure 2)

The isolation of SCAP is achieved by gentle separation of root apical papilla from the surface of the root with immature apex (i.e. located in the exterior of the root foramen area before the complete eruption of tooth in the oral cavity) during the extraction of third molars [47]. Root maturation results in the elimination of apical papilla; hence, the maturation period of teeth are important for isolation of SCAP. (Figure 2)

\subsubsection{Tissues contained periodontium-related stem cells (PDLSCS \& DFPCS) \& GSCs isolation}

PDLSC can be obtained from the middle third of the root surfaces of extracted PDL tissue, which is a soft connective tissue surrounded between the cementum and the inner wall of the alveolar bone socket. It's accomplished by scrapping surface of the middle third of the root [31].

DFPCs can be isolated by dissecting dental follicle from the upside of the dental crown from impacted teeth. Human dental follicle is an ectomesenchymal tissue that is derived from cranial neural crest. This tissue surrounds developing tooth germ and involves in the coordination of tooth eruption and periodontium formation. This tooth germ's tissue can easily be isolated after wisdom tooth extraction by routine orthodontical related surgeries. Impacted teeth, usually third molars, normally fail to erupt through the gum because of their encasement in the jawbone; therefore, routine surgical procedures are required for the extraction. [36, 37]. (Figure 2) 


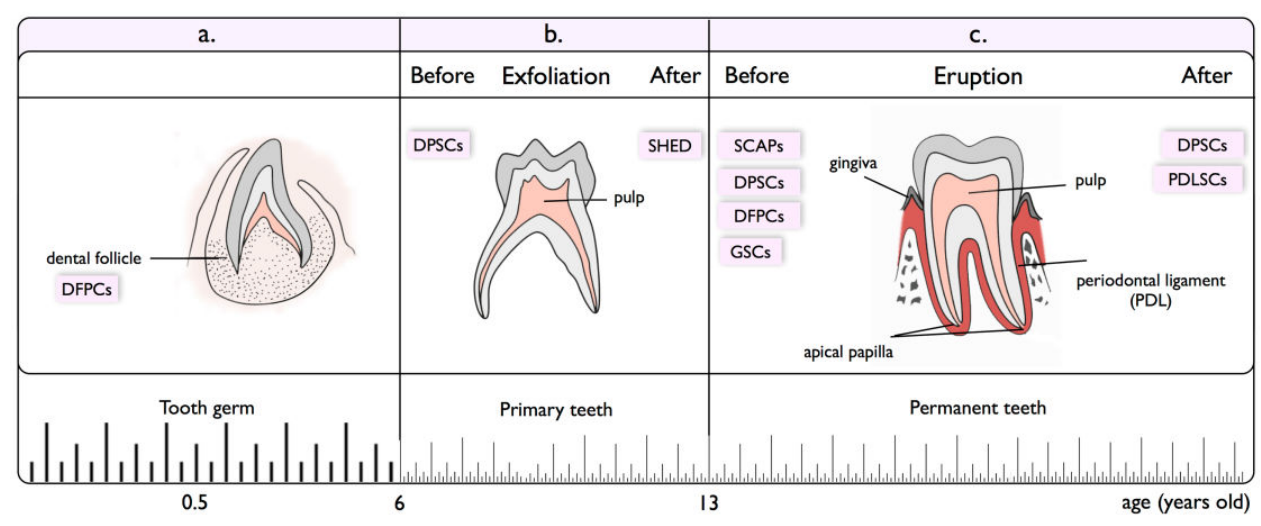

Figure 2. Overall view of dental-related stem cells based on different anatomical locations and stages during the human lifetime in. a. tooth germ, b. primary teeth, c. permanent teeth.

GSCs could be isolated from clinically healthy gingiva, which are obtained as remnant or discarded tissues following routine dental procedures [43]. (Figure 2)

\subsection{DSCs culture initiation}

In general, dental-related stem cells could isolate by either (1) enzymatic digestion (ED) of tissues or (2) outgrowth (OG) from tissue explant. In the case of enzymatic digestion, after the extraction, tissues are placed into the digestion enzymes, (generally, collagenase type-I \& dispase) for about $30-60$ minutes at $37^{\circ} \mathrm{C}$ to achieve single-cell suspensions. In order to purify DSCs, single-cell suspensions could be subjected into (1) size-sieved isolation (using $3 \mu \mathrm{m}$ strainer followed by $20 \mu \mathrm{m}$ strainer), (2) stem cell colony cultivation (single colony culture of stem cells) or (3) magnetic/ fluorescence activated cell sorting (sorting based on surface markers) [48].

In the outgrowth method (OG), tissues are minced into 1-2 $\mathrm{mm}$ pieces and placed into the culture dishes to outgrowth [18]. More recently, Lizier and his co-workers established a scaledup hIDPSCs culture system based on in vitro re-plating of pulp tissue explants followed by 3-4 days expansion [49].

There are some evidences, which suggested different behavior of DSCs according to the ED or OG isolation methods [41, 50,51]. According to Huang et al. DPSC isolated by ED method (DPSC-ED) from permanent teeth showed higher proliferation rate than those isolated by the OG method (DPSC-OG) [50]. Moreover, STRO-1 \& CD34 markers expressed more in DPSC-ED in comparison with DPSC-OG. DPSC-ED derived from deciduous and permanent teeth has been reported to display higher mineralization rate in the defined osteo/odonto medium [51, 52]. 


\subsection{DSCs culture systems}

Following the isolation of dental-related stem cells, the next step is to culture-expand the cells into the multiple copies since in the most strategies related to the cell-based-treatment of tissue defects, the copious amount of regenerating cells is needed. Many researchers have been focused on optimizing effective conditions under which DSCs can efficiently be propagated. On the other hand, differentiation potential of the multiplied cells must be determined because discovering the potential commitments of the cells may lead to better selection of them for future organ-targeted treatments $[27,48,53]$. Due to these considerations, this section opens up a brief overview for different DSC culture systems designed for the cell expansion and differentiation.

\subsubsection{Serum free vs. serum rich culture systems}

Normally, the isolation and expansion of DSC have depend on a high concentration of serum culture media (10\%), which provide better cell adhesion during the initial isolation of the cells. Unfortunately, in long-term cultivation, the high level of serum might lead to spontaneous differentiation or malignant transformation of cells. In addition, use of serum in culture may result in contamination of cell culture with bovine pathogen for instance bovine spongiform encephalopathy (BSE). For these reasons, serum free culture systems are highly recommended [54-57]. In this regards, many attempt has been made to optimize DSC cultivation in serumfree or low serum medium. For example, Karbanova et al. have reported that DPSCs cultivated in low-serum medium exhibit less proliferation rate and different expression of stem cell markers compared to those cultivated in serum rich medium [14]. In contrast, Hirata et al have cultivated the cells in serum free media and have found the same survival rate of the cells as those cultivated in the serum containing medium [58].

In the case of DFPCs, studies indicated that applying serum replacement media didn't affect the expression of connective tissue markers, such as collagen type I and type III, and also neural stem progenitor marker, nestin [59]; however, there is no information about the possible changes in other markers in this regards.

It's interesting to note that SHEDs \& PDLSCs cultivated in defined serum free media have been reported to display higher proliferation rate than those cultivated in the medium containing serum. Moreover such cells have found to express comparatively higher "stemness" markers [60]. Applying serum free media is one of the critical requirements for the future clinical treatments; therefore, additional works are needed for optimizing conditions to achieve final conclusion.

\subsubsection{Neurosphere-forming vs. adherent culture systems}

Sphere-forming culture systems are generally applied for neurogenic differentiation of stem cells. This culture system has also been used for DSC cultivation. The idea of applying such a system came from the consideration of neural crest origin of DSCs. It has been well established that neural stem/progenitor cells which are isolated from variety of sources are 
grown as neurospheres in defined serum-free culture medium supplemented with EGF and / or bFGF [61-63].

DSC Suspension culture was first suggested by Morita et al. (2007). These authors have cultivated PDLSCs in the sphere culture and found that PDLSC have the ability to form neurospheres in serum-free culture containing epidermal growth factor (EGF), basic fibroblast growth factor (bFGF) and leukemia inhibitory factor (LIF) with the ability to differentiate into both neural and mesodermal progeny [64].

In 2008, Sasaki et al. have cultivated DPSCs from the rat incisor in the sphere-culture and found that under these conditions, the cells expressed neural and glial markers. They have also noticed that CD81 positive DPSCs that were localized in odontoblast layer of apical portion of the dental pulp may have more potential to form neurospheres [65] Later on, it was demonstrated that sphere form of SCAP had multi-differentiation potential into mineralized cells, adipocytes and also myocytes under the defined media in vitro. Furthermore, In vivo studies have indicated that sphere SCAP showed more potential to generate mineralized tissues in comparison with the non-sphere SCAP [47].

\subsubsection{Co-culture systems}

Site-specific tissue interactions are essential for orchestration of proliferation, differentiation and also homeostasis of cells during the tooth germ development. In particular, epithelialmesenchymal interactions are the most important developmental events, which are involved in reciprocal crosstalk between the ectodermal and mesenchymal tissues. These sequential interactions are critical for tooth morphogenesis and cell differentiation [66]. To imitate these in vivo interactions, co-culture systems have been developed.

Using co-culture systems, some attempts have been made to promote DSCs differentiation in vitro. In this regards different cell types have been examined as co-culture cells. For example, it has been demonstrated that co-culture of DFPCs/SCAP could lead to formation of bone-like structure in vivo and in vitro. This occurs since interaction between the cells stimulate cementogenic/osteogenic differentiation of DFPCs leading to upregulation of bone intermediate proteins, such as bone morphogenetic protein 2 (BMP2), osteoprotegerin (OPG), bone sialoprotein (BSP) and osteocalcin (OCN), as well as downregulation of receptor activator of nuclear factor $\kappa B$ ligand (RANKL) [67]. In other experiment, Arakaki et al. have co-cultured DPSCs with dental epithelial cells and found that in such a system, dental epithelial cells secret BMP2 and BMP4, thereby promote odontoblastic differentiation of DPSCs [68]. Interestingly, it has also been reported that coculture of DPSCs with epithelial cells lead to epithelium invagination, as well [69]. Moreover, Wang et al. have established a co-culture of hDPSCs with osteoblasts and demonstrated that a higher mineralization and up-regulation of osteogenic-related genes in hDPSCs compared with those cultured in the absence of osteoblasts [70]. Furthermore, co-culture of DPSCs with non-dental MSCs, such as amniotic fluid-derived MSCs (AFMSCs), has been reported to leads to the bone differentiation of AF-MSCs indicating the pre-commitment of DPSCs to induce osteogenic differentiation [71]. 


\subsection{DSCs cryopreservation}

According to the diversity of dental-related stem cells and their remarkable features for cellmediated therapies and tissue engineering, developing a reliable method for cell banking have become a priority for future use. In this regard, cryopreservation could be established at the levels of teeth (organs), dental tissues or cells. In the case of teeth or dental tissues cryopreservation, minimal processing may needed for banking. There are some evidences which are demonstrated the successful cryopreservation of healthy and diseased teeth as well as dental tissues [72-78]. On the other hand, cryopreservation of DSCs still has been considered as an active area of the researches. There are several parameters which need to be considered prior to an establishment of a successful and more efficient protocol. The parameters which need to be determined include (1) DSCs-cultured passage(s), which leads to high-efficiency recovery post thaw, (2) concentration of cryoprotective agent, (3) cell concentration for high-efficient cryopreservation, (4) storage temperature, (5) the process of cryopreservation, and (6) evaluation of growth, surface markers and differentiation properties of DSCs after post thaw. So far, there have been many researches considering the comparative cryopreservation methods for optimizing the best protocols. More recently, it has been suggested that magnetic cryopreservation of DSCs was much better than conventional slow-freezing procedure in terms of cytotoxicity $[76,79,80]$.

\section{Dental-related stem cell based tissue regeneration}

Although DSCs are newborn in the field of stem cell therapy and tissue engineering, they have opened the promising windows not only in tooth repair and regeneration studies but also in other organs. To date, most of DSCs mediated cell therapies and tissue-engineering studies have been focused on the animal models. However, more recently, a few clinical trial studies have also been accomplished. Meanwhile, the immunogenicity of these cells should be more considered for their allogeneic transplantation.

\subsection{DSCs-based tooth engineering and regeneration of dental related tissue}

In general, in the field of dentistry, the final goal of tissue engineering is to develop toothreplacement therapies using the whole-bioengineered-tooth technology. To fulfill this, some authors have conducted the remarkable investigations. In this regard, there is some experiment in which either dissociated tooth germs or mesenchymal and epithelial cells derived from the tooth germs were prepared and loaded on to the prefabricated tooth-shaped scaffolds in order to fabricate a bioengineered tooth. Based on these investigations, tooth germ cells possessed a high potential to form dentin, enamel, pulp, and periodontal tissues in vivo [81-84]. However, using embryonic tooth germ and problems related to immunogenicity of animal transplantation, make this an unfeasible approach to clinical application of tooth regeneration [84].

Alternative cells would be DSCs. Using these cells, some preliminary studies have been accomplished to examine whether DSCs in combination with appropriate scaffold are able to promote regeneration of tooth tissues $[85,86]$. For instance, transplantation of PDLSCs 
loaded onto HA/TCP have been shown to result in the production of cementum in mice while using gelfoam (collagen based gelatin sponge) as the carrier has been reported to fail creating cementum [31, 87]. Sonoyama and co-workers have reported the creation of a root/periodontal complex being capable of supporting a porcelain crown in swine by applying both SCAP and PDLSCs in HA/TCP as a carrier [38]. In a study on canine model, we have recently succeeded to achieve regeneration on an experimentally-created defect in peridontium using autologous DPSCs loaded onto Bio-Oss scaffolds [88]. Furthermore, Nakashima et al. have reported a successful induction of whole-pulp regeneration after pulpectomy in a dog model using autologous DPSCs loaded onto 3D scaffold of collagenI \& II and SDF-1 (stromal-cell-derived factor-1) as the morphogene [89, 90]. Moreover, a culture system termed as organ-engineering methods using PDLSCs have been developed to generate not only tooth root, but also the surrounding periodontal tissues, including PDL and alveolar bone in mouse model [84]. In addition to the above-mentioned animal studies, there is a published retrospective pilot study in human. According to this trial, autologous transplantation of PDL stem/progenitor cells might provide therapeutic improvement for the periodontal defects without any adverse effects during 32-72 months of follow-up [91].

\subsection{DSCs-based tissue engineering and regeneration for other organs}

Apart from the potential applications in dental tissue engineering and therapy, DSCs have been opened a dynamic field in repair and regeneration of non-dental tissues. In this context, there are many investigations indicating applicability of dental related stem cells in variety of disease models in the animal. For instance, SHED has been reported to exhibit a potential to improve parkinson's disease in rat by differentiating into dopaminergic neuron-like cells. Based on the different investigations, these cells have also the ability to promote wound healing in mice [92-94]. It has been demonstrated that SHED also contribute to repair of the criticalsized calvarial defects in mice model [95].

Transplantation of DPSCs has been shown to improve alzheimer's and parkinson's disease as well as acute myocardial infarction in a rat model [96-98]. Similarly, transplantation of hIDPSCs in canine model with golden retriever muscular dystrophy (GRMD) resulted in some improvement [99]. In addition, it has been reported that transplantation of tissue-engineered hIDPSC sheet was successfully reconstructed the corneal epithelium in rabbits with total limbal stem cell deficiency (LSCD) [100]. Applying PDLSCs with collagen based gelatin sponge carrier have been found to improve facial wrinkles by generating large amounts of collagen fibers in the mouse indicating the potential capacity of PDLSC in the field of plastic surgery. In the case of GSCs, Intra-peritoneal (ip) administration of the cells displayed improvement of inflammation-related tissue destruction in experimental colitis [43].

\subsection{Immunogenicity of allogeneic transplantation of DSCs}

According to the promising place of DSCs in clinical treatments in future, considering the immunogenicity of DSC transplantation is critical. Although autologous DSCs rather than allogenous ones are preferred for repair and regeneration purposes, several limitations such 
as inadequate cell numbers and donor site morbidity carry out problems. Thus, more studies will be needed to evaluate immunogenicity of allogenous DSCs for the future clinical applications. There are some evidences suggested that DSCs, as the mesenchymal stem cells, have immunomodulatory properties both in vitro and in vivo. These studies are almost considering in vitro immunogenicity of DSCs by evaluating the expression of pro/anti-inflammatory mediators, such as MHC classes, TGF- $\beta$, ODO, HGF, nitric oxide (NO), prostaglandin, immunosuppressive minor $\mathrm{H}$ antigen (HLA-G), and interferon (IFN)- $\gamma$ as well as effect of DSCs in suppression of T cells proliferation [28, 101, 102].

In addition, in vivo studies also showed DSCs immunomodulatory functions. For instance, SHED transplantation up-regulated the ratio of Treg (regulatory T cells) and Th17 in MRL/ lpr mice model compared to the BM-MSCs; meanwhile, no change in the expression of IL- 6 \& IL-10 was detected. On the other hand, transplantation of human DPSCs into the rat model didn't initiate the immunologic responses in recipients [103]. Moreover, in another study, it was demonstrated that transplantation of hIDPSCs didn't show the immune reaction in the canine [99].

In spite of existing evidences, which suggested the immunomodulatory effect of DSCs, there are some data proposing that there is different expression of immune receptors, such as tolllike receptors (TLRs) on the cells. Presence of this receptor could affect the immumomodulatory phenotypes of DPSCs and DFPCs [101, 104, 105]; On the other hand, since some studies suggested that BM-MSCs may regain immunogenic property upon differentiation [106], the subject of immunogenicity of DSCs must be examined not only in an undifferentiated state but also in differentiated form.

\section{Conclusion}

Dental-related tissue contained several types of stem cells collectively referred to as dental stem cells (DSCs). In this stem cell family, there are 6 named member so far recognized and described. These include dental pulp stem cell (DPSCs), stem cells from human exfoliated deciduous teeth (SHED), periodontal ligament stem cells (PDLSCs), stem cells from apical papilla (SCAP) of developing tooth, dental follicle stem/progenitor cells (DFPCs) and gingival stem cells (GSCs). All these easily-accessible stem cells can be derived from dental tissue obtained from both young and adult patients. Furthermore the cells are described as having immunomodulatory function. These characteristics make DSCs a unique source for repair and regeneration of injured tissue. In this context, many studies have so far been conducted on the animal models and the reports together indicated the extensive potential of the cells in tissue repair and regeneration not only in teeth but also in other organs. After all these animal studies, some centers have started clinical trials to examine the cell potential in human diseases. To exploit the extensive regenerating potential of DSCs in clinic, many additional clinical trials must be planed and conducted till therapeutic protocols using these cells become established. 


\section{Author details}

Razieh Karamzadeh and Mohamadreza Baghaban Eslaminejad*

*Address all correspondence to: eslami@royaninstitute.org; and bagesla@yahoo.com

Department of Stem Cell and Developmental Biology, Cell Science Research Center, Royan Institute for Stem Cell Biology and Technology, ACECR, Tehran, Iran

\section{References}

[1] Morsczeck C, Schmalz G, Reichert TE, Vollner F, Galler K, Driemel O. Somatic Stem Cells s for regenerative dentistry. Clin Oral Investig. 2008 Jun;12(2):113-8.

[2] Huang GT, Gronthos S, Shi S. Mesenchymal stem cells derived from dental tissues vs. those from other sources: their biology and role in regenerative medicine. J Dent Res. 2009 Sep;88(9):792-806.

[3] Zhang QZ, Nguyen AL, Yu WH, Le AD. Human oral mucosa and gingiva: a unique reservoir for mesenchymal stem cells. J Dent Res. 2012 Nov;91(11):1011-8.

[4] Ferro F, Spelat R, D'Aurizio F, Puppato E, Pandolfi M, Beltrami AP, et al. Dental Pulp Stem Cells Differentiation Reveals New Insights in Oct4A Dynamics. PLoS One. 2012;7(7):e41774.

[5] Rodriguez-Lozano FJ, Bueno C, Insausti CL, Meseguer L, Ramirez MC, Blanquer M, et al. Mesenchymal stem cells derived from dental tissues. Int Endod J. 2011 Sep; 44(9):800-6.

[6] Yamamura T. Differentiation of pulpal cells and inductive influences of various matrices with reference to pulpal wound healing. J Dent Res. 1985 Apr;64 Spec No: $530-40$.

[7] Gronthos S, Mankani M, Brahim J, Robey PG, Shi S. Postnatal human dental pulp stem cells (DPSCs) in vitro and in vivo. Proc Natl Acad Sci U S A. 2000 Dec 5;97(25): 13625-30.

[8] Tandon S, Saha R, Rajendran R, Nayak R. Dental pulp stem cells from primary and permanent teeth: quality analysis. J Clin Pediatr Dent. 2010 Fall;35(1):53-8.

[9] Mitsiadis TA, Feki A, Papaccio G, Caton J. Dental pulp stem cells, niches, and notch signaling in tooth injury. Adv Dent Res. 2011 Jul;23(3):275-9.

[10] Shi S, Gronthos S. Perivascular niche of postnatal mesenchymal stem cells in human bone marrow and dental pulp. J Bone Miner Res. 2003 Apr;18(4):696-704. 
[11] Eslaminejad MB, Vahabi S, Shariati M, Nazarian H. In vitro Growth and Characterization of Stem Cells from Human Dental Pulp of Deciduous Versus Permanent Teeth. J Dent (Tehran). 2010 Fall;7(4):185-95.

[12] Huang AH, Chen YK, Lin LM, Shieh TY, Chan AW. Isolation and characterization of dental pulp stem cells from a supernumerary tooth. J Oral Pathol Med. 2008 Oct; 37(9):571-4.

[13] Liu L, Wei X, Ling J, Wu L, Xiao Y. Expression pattern of Oct-4, Sox2, and c-Myc in the primary culture of human dental pulp derived cells. J Endod. 2011 Apr;37(4): 466-72.

[14] Karbanova J, Soukup T, Suchanek J, Pytlik R, Corbeil D, Mokry J. Characterization of dental pulp stem cells from impacted third molars cultured in low serum-containing medium. Cells Tissues Organs. 2011;193(6):344-65.

[15] Gronthos S, Brahim J, Li W, Fisher LW, Cherman N, Boyde A, et al. Stem cell properties of human dental pulp stem cells. J Dent Res. 2002 Aug;81(8):531-5.

[16] Shi S, Bartold PM, Miura M, Seo BM, Robey PG, Gronthos S. The efficacy of mesenchymal stem cells to regenerate and repair dental structures. Orthod Craniofac Res. 2005 Aug;8(3):191-9.

[17] Pierdomenico L, Bonsi L, Calvitti M, Rondelli D, Arpinati M, Chirumbolo G, et al. Multipotent mesenchymal stem cells with immunosuppressive activity can be easily isolated from dental pulp. Transplantation. 2005 Sep 27;80(6):836-42.

[18] Kerkis I, Kerkis A, Dozortsev D, Stukart-Parsons GC, Gomes Massironi SM, Pereira $\mathrm{LV}$, et al. Isolation and characterization of a population of immature dental pulp stem cells expressing OCT-4 and other embryonic stem cell markers. Cells Tissues Organs. 2006;184(3-4):105-16.

[19] Siqueira da Fonseca SA, Abdelmassih S, de Mello Cintra Lavagnolli T, Serafim RC, Clemente Santos EJ, Mota Mendes C, et al. Human immature dental pulp stem cells' contribution to developing mouse embryos: production of human/mouse preterm chimaeras. Cell Prolif. 2009 Apr;42(2):132-40.

[20] Beltrao-Braga PI, Pignatari GC, Maiorka PC, Oliveira NA, Lizier NF, Wenceslau CV, et al. Feeder-free derivation of induced pluripotent stem cells from human immature dental pulp stem cells. Cell Transplant. 2011 Apr 1.

[21] Miura M, Gronthos S, Zhao M, Lu B, Fisher LW, Robey PG, et al. SHED: stem cells from human exfoliated deciduous teeth. Proc Natl Acad Sci U S A. 2003 May 13;100(10):5807-12.

[22] Nakamura S, Yamada Y, Katagiri W, Sugito T, Ito K, Ueda M. Stem cell proliferation pathways comparison between human exfoliated deciduous teeth and dental pulp stem cells by gene expression profile from promising dental pulp. J Endod. 2009 Nov;35(11):1536-42. 
[23] Nourbakhsh N, Soleimani M, Taghipour Z, Karbalaie K, Mousavi SB, Talebi A, et al. Induced in vitro differentiation of neural-like cells from human exfoliated deciduous teeth-derived stem cells. Int J Dev Biol. 2011;55(2):189-95.

[24] Chadipiralla K, Yochim JM, Bahuleyan B, Huang CY, Garcia-Godoy F, Murray PE, et al. Osteogenic differentiation of stem cells derived from human periodontal ligaments and pulp of human exfoliated deciduous teeth. Cell Tissue Res. 2010 May; 340(2):323-33.

[25] Sakai VT, Zhang Z, Dong Z, Neiva KG, Machado MA, Shi S, et al. SHED differentiate into functional odontoblasts and endothelium. J Dent Res. 2010 Aug;89(8):791-6.

[26] Yamada Y, Nakamura S, Ito K, Sugito T, Yoshimi R, Nagasaka T, et al. A feasibility of useful cell-based therapy by bone regeneration with deciduous tooth stem cells, dental pulp stem cells, or bone-marrow-derived mesenchymal stem cells for clinical study using tissue engineering technology. Tissue Eng Part A. 2010 Jun;16(6): 1891-900.

[27] Morsczeck C, Vollner F, Saugspier M, Brandl C, Reichert TE, Driemel O, et al. Comparison of human dental follicle cells (DFCs) and stem cells from human exfoliated deciduous teeth (SHED) after neural differentiation in vitro. Clin Oral Investig. 2010 Aug;14(4):433-40.

[28] Yamaza T, Kentaro A, Chen C, Liu Y, Shi Y, Gronthos S, et al. Immunomodulatory properties of stem cells from human exfoliated deciduous teeth. Stem Cell Res Ther. 2010;1(1):5.

[29] Zheng Y, Liu Y, Zhang CM, Zhang HY, Li WH, Shi S, et al. Stem cells from deciduous tooth repair mandibular defect in swine. J Dent Res. 2009 Mar;88(3):249-54.

[30] Monteiro BG, Serafim RC, Melo GB, Silva MC, Lizier NF, Maranduba CM, et al. Human immature dental pulp stem cells share key characteristic features with limbal stem cells. Cell Prolif. 2009 Oct;42(5):587-94.

[31] Seo BM, Miura M, Gronthos S, Bartold PM, Batouli S, Brahim J, et al. Investigation of multipotent postnatal stem cells from human periodontal ligament. Lancet. $2004 \mathrm{Jul}$ 10-16;364(9429):149-55.

[32] Wada N, Menicanin D, Shi S, Bartold PM, Gronthos S. Immunomodulatory properties of human periodontal ligament stem cells. J Cell Physiol. 2009 Jun;219(3):667-76.

[33] Trubiani O, Zalzal SF, Paganelli R, Marchisio M, Giancola R, Pizzicannella J, et al. Expression profile of the embryonic markers nanog, OCT-4, SSEA-1, SSEA-4, and frizzled-9 receptor in human periodontal ligament mesenchymal stem cells. J Cell Physiol. 2010 Oct;225(1):123-31.

[34] Kawanabe N, Murata S, Murakami K, Ishihara Y, Hayano S, Kurosaka H, et al. Isolation of multipotent stem cells in human periodontal ligament using stage-specific embryonic antigen-4. Differentiation. 2010 Feb;79(2):74-83. 
[35] Gay IC, Chen S, MacDougall M. Isolation and characterization of multipotent human periodontal ligament stem cells. Orthod Craniofac Res. 2007 Aug;10(3):149-60.

[36] Morsczeck C, Gotz W, Schierholz J, Zeilhofer F, Kuhn U, Mohl C, et al. Isolation of precursor cells (PCs) from human dental follicle of wisdom teeth. Matrix Biol. 2005 Apr;24(2):155-65.

[37] Kemoun P, Laurencin-Dalicieux S, Rue J, Farges JC, Gennero I, Conte-Auriol F, et al. Human dental follicle cells acquire cementoblast features under stimulation by BMP-2/-7 and enamel matrix derivatives (EMD) in vitro. Cell Tissue Res. 2007 Aug; 329(2):283-94.

[38] Sonoyama W, Liu Y, Fang D, Yamaza T, Seo BM, Zhang C, et al. Mesenchymal stem cell-mediated functional tooth regeneration in swine. PLoS One. 2006;1:e79.

[39] Sonoyama W, Liu Y, Yamaza T, Tuan RS, Wang S, Shi S, et al. Characterization of the apical papilla and its residing stem cells from human immature permanent teeth: a pilot study. J Endod. 2008 Feb;34(2):166-71.

[40] Abe S, Yamaguchi S, Watanabe A, Hamada K, Amagasa T. Hard tissue regeneration capacity of apical pulp derived cells (APDCs) from human tooth with immature apex. Biochem Biophys Res Commun. 2008 Jun 20;371(1):90-3.

[41] Bakopoulou A, Leyhausen G, Volk J, Tsiftsoglou A, Garefis P, Koidis P, et al. Comparative analysis of in vitro osteo/odontogenic differentiation potential of human dental pulp stem cells (DPSCs) and stem cells from the apical papilla (SCAP). Arch Oral Biol. 2011 Jul;56(7):709-21.

[42] Ding G, Liu Y, An Y, Zhang C, Shi S, Wang W, et al. Suppression of T cell proliferation by root apical papilla stem cells in vitro. Cells Tissues Organs. 2010;191(5): 357-64.

[43] Zhang Q, Shi S, Liu Y, Uyanne J, Shi Y, Le AD. Mesenchymal stem cells derived from human gingiva are capable of immunomodulatory functions and ameliorate inflammation-related tissue destruction in experimental colitis. J Immunol. 2009 Dec 15;183(12):7787-98.

[44] Tang L, Li N, Xie H, Jin Y. Characterization of mesenchymal stem cells from human normal and hyperplastic gingiva. J Cell Physiol. 2011 Mar;226(3):832-42.

[45] Tomar GB, Srivastava RK, Gupta N, Barhanpurkar AP, Pote ST, Jhaveri HM, et al. Human gingiva-derived mesenchymal stem cells are superior to bone marrow-derived mesenchymal stem cells for cell therapy in regenerative medicine. Biochem Biophys Res Commun. 2010 Mar 12;393(3):377-83.

[46] Alongi DJ, Yamaza T, Song Y, Fouad AF, Romberg EE, Shi S, et al. Stem/progenitor cells from inflamed human dental pulp retain tissue regeneration potential. Regen Med. 2010 Jul;5(4):617-31. 
[47] Abe S, Hamada K, Yamaguchi S, Amagasa T, Miura M. Characterization of the radioresponse of human apical papilla-derived cells. Stem Cell Res Ther. 2011;2(1):2.

[48] Yan M, Yu Y, Zhang G, Tang C, Yu J. A journey from dental pulp stem cells to a biotooth. Stem Cell Rev. 2011 Mar;7(1):161-71.

[49] Lizier NF, Kerkis A, Gomes CM, Hebling J, Oliveira CF, Caplan AI, et al. Scaling-up of dental pulp stem cells isolated from multiple niches. PLoS One. 2012;7(6):e39885.

[50] Huang GT, Sonoyama W, Chen J, Park SH. In vitro characterization of human dental pulp cells: various isolation methods and culturing environments. Cell Tissue Res. 2006 May;324(2):225-36.

[51] Bakopoulou A, Leyhausen G, Volk J, Tsiftsoglou A, Garefis P, Koidis P, et al. Assessment of the impact of two different isolation methods on the osteo/odontogenic differentiation potential of human dental stem cells derived from deciduous teeth. Calcif Tissue Int. 2011 Feb;88(2):130-41.

[52] Karamzadeh R, Eslaminejad MB, Aflatoonian R. Isolation, characterization and comparative differentiation of human dental pulp stem cells derived from permanent teeth by using two different methods. J Vis Exp. 2012(69).

[53] Kim SH, Kim YS, Lee SY, Kim KH, Lee YM, Kim WK, et al. Gene expression profile in mesenchymal stem cells derived from dental tissues and bone marrow. J Periodontal Implant Sci. 2011 Aug;41(4):192-200.

[54] Tseng PY, Chen CJ, Sheu CC, Yu CW, Huang YS. Spontaneous differentiation of adult rat marrow stromal cells in a long-term culture. J Vet Med Sci. 2007 Feb;69(2): 95-102.

[55] Torsvik A, Rosland GV, Svendsen A, Molven A, Immervoll H, McCormack E, et al. Spontaneous malignant transformation of human mesenchymal stem cells reflects cross-contamination: putting the research field on track - letter. Cancer Res. 2010 Aug 1;70(15):6393-6.

[56] Gou S, Wang C, Liu T, Wu H, Xiong J, Zhou F, et al. Spontaneous differentiation of murine bone marrow-derived mesenchymal stem cells into adipocytes without malignant transformation after long-term culture. Cells Tissues Organs. 2010;191(3): 185-92.

[57] Ren Z, Wang J, Zhu W, Guan Y, Zou C, Chen Z, et al. Spontaneous transformation of adult mesenchymal stem cells from cynomolgus macaques in vitro. Exp Cell Res. 2011 Dec 10;317(20):2950-7.

[58] Hirata TM, Ishkitiev N, Yaegaki K, Calenic B, Ishikawa H, Nakahara T, et al. Expression of multiple stem cell markers in dental pulp cells cultured in serum-free media. J Endod. 2010 Jul;36(7):1139-44. 
[59] Morsczeck C, Ernst W, Florian C, Reichert TE, Proff P, Bauer R, et al. Gene expression of nestin, collagen type I and type III in human dental follicle cells after cultivation in serum-free medium. Oral Maxillofac Surg. 2008 Jul;12(2):89-92.

[60] Tarle SA, Shi S, Kaigler D. Development of a serum-free system to expand dental-derived stem cells: PDLSCs and SHEDs. J Cell Physiol. 2011 Jan;226(1):66-73.

[61] Reynolds BA, Weiss S. Generation of neurons and astrocytes from isolated cells of the adult mammalian central nervous system. Science. 1992 Mar 27;255(5052): 1707-10.

[62] Kopen GC, Prockop DJ, Phinney DG. Marrow stromal cells migrate throughout forebrain and cerebellum, and they differentiate into astrocytes after injection into neonatal mouse brains. Proc Natl Acad Sci U S A. 1999 Sep 14;96(19):10711-6.

[63] Tropepe V, Coles BL, Chiasson BJ, Horsford DJ, Elia AJ, McInnes RR, et al. Retinal stem cells in the adult mammalian eye. Science. 2000 Mar 17;287(5460):2032-6.

[64] Techawattanawisal W, Nakahama K, Komaki M, Abe M, Takagi Y, Morita I. Isolation of multipotent stem cells from adult rat periodontal ligament by neurosphere-forming culture system. Biochem Biophys Res Commun. 2007 Jun 15;357(4):917-23.

[65] Sasaki R, Aoki S, Yamato M, Uchiyama H, Wada K, Okano T, et al. Neurosphere generation from dental pulp of adult rat incisor. Eur J Neurosci. 2008 Feb;27(3):538-48.

[66] Hargreaves KMaG, Harold E. Seltzer and Bender's Dental Pulp. 2002 ed: Quintessence Pub. Co., ; 2002. p. 500 pages.

[67] Bai Y, Matsuzaka K, Hashimoto S, Kokubu E, Wang X, Inoue T. Formation of bonelike tissue by dental follicle cells co-cultured with dental papilla cells. Cell Tissue Res. 2010 Nov;342(2):221-31.

[68] Arakaki M, Ishikawa M, Nakamura T, Iwamoto T, Yamada A, Fukumoto E, et al. Role of epithelial-stem cell interactions during dental cell differentiation. J Biol Chem. 2012 Mar 23;287(13):10590-601.

[69] Xiao L, Tsutsui T. Three-dimensional epithelial and mesenchymal cell co-cultures form early tooth epithelium invagination-like structures: expression patterns of relevant molecules. J Cell Biochem. 2012 Jun;113(6):1875-85.

[70] Wang Y, Yao J, Yuan M, Zhang Z, Hu W. Osteoblasts can induce dental pulp stem cells to undergo osteogenic differentiation. Cytotechnology. 2012 Jul 18.

[71] De Rosa A, Tirino V, Paino F, Tartaglione A, Mitsiadis T, Feki A, et al. Amniotic fluid-derived mesenchymal stem cells lead to bone differentiation when cocultured with dental pulp stem cells. Tissue Eng Part A. 2011 Mar;17(5-6):645-53.

[72] Chen YK, Huang AH, Chan AW, Shieh TY, Lin LM. Human dental pulp stem cells derived from different cryopreservation methods of human dental pulp tissues of diseased teeth. J Oral Pathol Med. 2011 Nov;40(10):793-800. 
[73] Perry BC, Zhou D, Wu X, Yang FC, Byers MA, Chu TM, et al. Collection, cryopreservation, and characterization of human dental pulp-derived mesenchymal stem cells for banking and clinical use. Tissue Eng Part C Methods. 2008 Jun;14(2):149-56.

[74] Woods EJ, Perry BC, Hockema JJ, Larson L, Zhou D, Goebel WS. Optimized cryopreservation method for human dental pulp-derived stem cells and their tissues of origin for banking and clinical use. Cryobiology. 2009 Oct;59(2):150-7.

[75] Temmerman L, Beele H, Dermaut LR, Van Maele G, De Pauw GA. Influence of cryopreservation on the pulpal tissue of immature third molars in vitro. Cell Tissue Bank. 2010 Aug;11(3):281-9.

[76] Lee SY, Chiang PC, Tsai YH, Tsai SY, Jeng JH, Kawata T, et al. Effects of cryopreservation of intact teeth on the isolated dental pulp stem cells. J Endod. 2010 Aug;36(8): 1336-40.

[77] Seo BM, Miura M, Sonoyama W, Coppe C, Stanyon R, Shi S. Recovery of stem cells from cryopreserved periodontal ligament. J Dent Res. 2005 Oct;84(10):907-12.

[78] Gioventu S, Andriolo G, Bonino F, Frasca S, Lazzari L, Montelatici E, et al. A novel method for banking dental pulp stem cells. Transfus Apher Sci. 2012 Jul 11.

[79] Kaku M, Kamada H, Kawata T, Koseki H, Abedini S, Kojima S, et al. Cryopreservation of periodontal ligament cells with magnetic field for tooth banking. Cryobiology. 2010 Aug;61(1):73-8.

[80] Lee SY, Huang GW, Shiung JN, Huang YH, Jeng JH, Kuo TF, et al. Magnetic cryopreservation for dental pulp stem cells. Cells Tissues Organs. 2012;196(1):23-33.

[81] Yen AH, Sharpe PT. Stem cells and tooth tissue engineering. Cell Tissue Res. 2008 Jan;331(1):359-72.

[82] Oshima M, Mizuno M, Imamura A, Ogawa M, Yasukawa M, Yamazaki H, et al. Functional tooth regeneration using a bioengineered tooth unit as a mature organ replacement regenerative therapy. PLoS One. 2011;6(7):e21531.

[83] Wang Y, Preston B, Guan G. Tooth bioengineering leads the next generation of dentistry. Int J Paediatr Dent. 2012 Jan 8.

[84] Nakahara T. Potential feasibility of dental stem cells for regenerative therapies: stem cell transplantation and whole-tooth engineering. Odontology. 2011 Jul;99(2):105-11.

[85] Mantesso A, Sharpe P. Dental stem cells for tooth regeneration and repair. Expert Opin Biol Ther. 2009 Sep;9(9):1143-54.

[86] Nakashima M, Akamine A. The application of tissue engineering to regeneration of pulp and dentin in endodontics. J Endod. 2005 Oct;31(10):711-8. 
[87] Fang D, Seo BM, Liu Y, Sonoyama W, Yamaza T, Zhang C, et al. Transplantation of mesenchymal stem cells is an optimal approach for plastic surgery. Stem Cells. 2007 Apr;25(4):1021-8.

[88] Eslaminejad BM, Khorsand A, Arabsolghar M, Paknejad M, Ghaedi B, Rokn AR, et al. Autologous Dental Pulp Stem Cells in Regeneration of Defect Created in Canine Periodontal Tissue. J Oral Implantol. 2012 Aug 1.

[89] Nakashima M, Iohara K. Regeneration of dental pulp by stem cells. Adv Dent Res. 2011 Jul;23(3):313-9.

[90] Iohara K, Imabayashi K, Ishizaka R, Watanabe A, Nabekura J, Ito M, et al. Complete pulp regeneration after pulpectomy by transplantation of CD105+ stem cells with stromal cell-derived factor-1. Tissue Eng Part A. 2011 Aug;17(15-16):1911-20.

[91] Feng F, Akiyama K, Liu Y, Yamaza T, Wang TM, Chen JH, et al. Utility of PDL progenitors for in vivo tissue regeneration: a report of 3 cases. Oral Dis. 2010 Jan;16(1): 20-8.

[92] Wang J, Wang X, Sun Z, Yang H, Shi S, Wang S. Stem cells from human-exfoliated deciduous teeth can differentiate into dopaminergic neuron-like cells. Stem Cells Dev. 2010 Sep;19(9):1375-83.

[93] Nishino Y, Yamada Y, Ebisawa K, Nakamura S, Okabe K, Umemura E, et al. Stem cells from human exfoliated deciduous teeth (SHED) enhance wound healing and the possibility of novel cell therapy. Cytotherapy. 2011 May;13(5):598-605.

[94] Nishino Y, Ebisawa K, Yamada Y, Okabe K, Kamei Y, Ueda M. Human deciduous teeth dental pulp cells with basic fibroblast growth factor enhance wound healing of skin defect. J Craniofac Surg. 2011 Mar;22(2):438-42.

[95] Seo BM, Sonoyama W, Yamaza T, Coppe C, Kikuiri T, Akiyama K, et al. SHED repair critical-size calvarial defects in mice. Oral Dis. 2008 Jul;14(5):428-34.

[96] Apel C, Forlenza OV, de Paula VJ, Talib LL, Denecke B, Eduardo CP, et al. The neuroprotective effect of dental pulp cells in models of Alzheimer's and Parkinson's disease. J Neural Transm. 2009 Jan;116(1):71-8.

[97] Nesti C, Pardini C, Barachini S, D'Alessandro D, Siciliano G, Murri L, et al. Human dental pulp stem cells protect mouse dopaminergic neurons against MPP+ or rotenone. Brain Res. 2011 Jan 7;1367:94-102.

[98] Gandia C, Arminan A, Garcia-Verdugo JM, Lledo E, Ruiz A, Minana MD, et al. Human dental pulp stem cells improve left ventricular function, induce angiogenesis, and reduce infarct size in rats with acute myocardial infarction. Stem Cells. 2008 Mar; 26(3):638-45.

[99] Kerkis I, Ambrosio CE, Kerkis A, Martins DS, Zucconi E, Fonseca SA, et al. Early transplantation of human immature dental pulp stem cells from baby teeth to golden 
retriever muscular dystrophy (GRMD) dogs: Local or systemic? J Transl Med. 2008;6:35.

[100] Gomes JA, Geraldes Monteiro B, Melo GB, Smith RL, Cavenaghi Pereira da Silva M, Lizier NF, et al. Corneal reconstruction with tissue-engineered cell sheets composed of human immature dental pulp stem cells. Invest Ophthalmol Vis Sci. 2010 Mar; 51(3):1408-14.

[101] Tomic S, Djokic J, Vasilijic S, Vucevic D, Todorovic V, Supic G, et al. Immunomodulatory properties of mesenchymal stem cells derived from dental pulp and dental follicle are susceptible to activation by toll-like receptor agonists. Stem Cells Dev. 2011 Apr;20(4):695-708.

[102] Bifari F, Pacelli L, Krampera M. Immunological properties of embryonic and adult stem cells. World J Stem Cells. 2010 Jun 26;2(3):50-60.

[103] de Mendonca Costa A, Bueno DF, Martins MT, Kerkis I, Kerkis A, Fanganiello RD, et al. Reconstruction of large cranial defects in nonimmunosuppressed experimental design with human dental pulp stem cells. J Craniofac Surg. 2008 Jan;19(1):204-10.

[104] Waterman RS, Tomchuck SL, Henkle SL, Betancourt AM. A new mesenchymal stem cell (MSC) paradigm: polarization into a pro-inflammatory MSC1 or an Immunosuppressive MSC2 phenotype. PLoS One. 2010;5(4):e10088.

[105] English K, Mahon BP. Allogeneic mesenchymal stem cells: agents of immune modulation. J Cell Biochem. 2011 Aug;112(8):1963-8.

[106] Liu H, Kemeny DM, Heng BC, Ouyang HW, Melendez AJ, Cao T. The immunogenicity and immunomodulatory function of osteogenic cells differentiated from mesenchymal stem cells. J Immunol. 2006 Mar 1;176(5):2864-71. 